\title{
Entre vote et marchandage
}

Partis nationaux et groupes ethniques aux élections parlementaires de 1908

Between election and bargain: national parties and ethnics groups in the 1908 general election

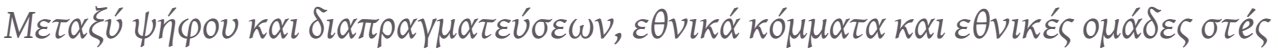

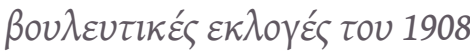

\section{Tasos Kostopoulos}

\section{OpenEdition}

\section{Journals}

Édition électronique

URL : https://journals.openedition.org/ceb/1269

DOI : $10.4000 /$ ceb. 1269

ISSN : 2261-4184

Éditeur

INALCO

\section{Édition imprimée}

Date de publication : 9 janvier 2012

ISSN : 0290-7402

\section{Référence électronique}

Tasos Kostopoulos, «Entre vote et marchandage », Cahiers balkaniques [En ligne], 40 | 2012, mis en ligne le 09 mars 2012, consulté le 06 juillet 2021. URL : http://journals.openedition.org/ceb/1269 ; DOI : https://doi.org/10.4000/ceb.1269

Ce document a été généré automatiquement le 6 juillet 2021.

Cahiers balkaniques est mis à disposition selon les termes de la Licence Creative Commons Attribution - Pas d'Utilisation Commerciale 4.0 International. 


\section{Entre vote et marchandage}

Partis nationaux et groupes ethniques aux élections parlementaires de 1908

Between election and bargain: national parties and ethnics groups in the 1908 general election

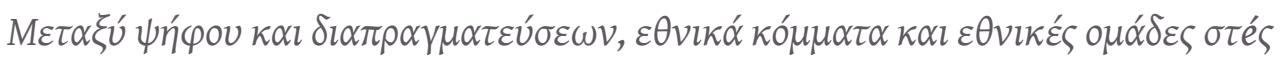

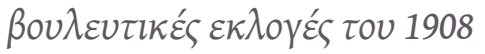

Tasos Kostopoulos

1 Les élections parlementaires de 1908 ont été la première expérience de cette sorte pour la population ottomane (et aussi pour une bonne partie des citoyens futurs des États Balkaniques voisins). Même si l'élection des notables à l'échelle locale (muhtars des villages ou des mahallas, épitropes de l'église, chefs des esnafs, etc.) était une pratique courante dans la société ottomane, la désignation des dignitaires supérieurs était toujours réservée à la volonté pure et simple de la Sublime Porte ; quant aux membres des premiers Parlements ottomans de 1876-1877, à l'époque de Tanzimat, ils étaient simplement nommés par les conseils administratifs déjà existants au niveau du kaza, du sandjak et du vilayet ${ }^{1}$.

2 Ces mêmes élections ont été la première épreuve réelle de la crédibilité des promesses faites par la révolution Jeune-Turque, d'instaurer un régime d'égalité entre les composantes de la mosaïque ethno-nationale de l'Empire. Elles ont été aussi un test de l'adaptabilité des partis nationaux et de leurs appareils dirigeants, de leur capacité à fonctionner sous des conditions radicalement différentes de celles de la lutte armée des années précédentes.

3 Jusqu'à présent, les historiographies de la Grèce et de la République de la Macédoine ex-yougoslave ont plus ou moins évité ce sujet ${ }^{2}$. En ce qui concerne l'historiographie bulgare, on peut se référer à un article publié il y a 35 ans qui, malheureusement, reproduit dans une grande mesure les clichés traditionnels nationalistes sur les « injustices historiques » subies par la nationalité bulgare prépondérante dans presque toute la région convoitée ${ }^{3}$. Dans l'article que vous allez lire, on va décrire la confrontation électorale de 1908 dans la Macédoine ottomane, comme un jeu politique 
entre différents mouvements et centres politiques rivaux d'obédience nationale ; on va examiner leurs stratégies électorales, en utilisant principalement la correspondance diplomatique inédite des consulats de la Grèce et de la Bulgarie, accessible aujourd'hui aux archives du Ministère grec des Affaires extérieures (IAYE) et aux Archives centrales d'État bulgare (цДА).

\section{Partis nationaux et politiques}

Une des spécificités les plus marquantes du conflit macédonien pendant les décennies précédant le Hürriyet, a été la formation de véritables " partis » politiques d'obédience nationale, organisés autour des métropoles et des consulats respectifs, mais jouissant d'une autonomie assez considérable en ce qui concernait les affaires locales ${ }^{4}$. À l'intérieur de chaque groupe ethnolinguistique, on pouvait ainsi distinguer plusieurs "partis", se réclamant l'hégémonie au niveau local ou / et régional. Les Slaves Macédoniens étaient partagés entre les partis grec, bulgare, serbe et - après 1904 macédonien - (ce dernier regroupé autour de la faction autonomiste de l'ORIM (Organisation révolutionnaire intérieure macédonienne), qui a pris des distances envers la Bulgarie officielle sans pour autant avoir définitivement coupé ses références à la communauté imaginaire de la nationalité bulgare ${ }^{5}$. Les Valaques étaient partagés entre les partis grec et roumain, les chrétiens albanophones entre les partis grec et albanais ; après 1908, les Albanais musulmans ont été aussi divisés entre les partis turc et albanais ${ }^{6}$. Grâce à leur identification complète avec la classe dominante ottomane et l'évolution nationale hellénique du Millet-i-Rum, les musulmans turcophones et les chrétiens grécophones étaient les seuls à ne pas se confronter à pareille division "nationale»; les communautés grécophones ont pourtant connu pas mal de conflits politiques internes, dont la forme la plus répandue était celle de la confrontation entre «le parti du métropolite » local et le parti de ses adversaires ${ }^{7}$. Une troisième catégorie des groupes ethniques englobait les chrétiens turcophones de la région de Zihna, dans le sandjak de Serrès, ainsi que les musulmans grécophones de Pinde (Valaades) et slavophones (Pomaks et Torbesh): dans ces cas-là, aucun mouvement nationaliste n'a revendiqué la loyauté des populations en cause, et leur incorporation dans les communautés imaginaires nationales dominantes dans les millet respectifs ne fut alors en aucune manière perturbée.

La structure intérieure des partis nationaux ressemblait à celle des partis politiques ordinaires : on pouvait y discerner un noyau dur des adeptes convaincus, entouré des strates concentriques de partisans moins fervents ou d'effectifs flottants, passant d'un parti national à l'autre en suivant les changements de la corrélation des forces sur le terrain. L'appartenance partisane, dite "nationale ", pouvait être déterminée à la fois par des facteurs idéologiques et politiques liés à de grands projets rivaux pour l'avenir collectif (dedans ou dehors l'Empire), aussi bien que par des contradictions sociales ou personnelles de caractère purement local.

Mise en question par l'émergence du mouvement révolutionnaire macédonien, luimême divisé entre la représentation d'un irrédentisme bulgare ultranationaliste et une tendance "macédoniste» de plus en plus méfiante envers les nationalismes rivaux revendiquant la loyauté de la population slave macédonienne, la domination des partis nationaux sur les communautés chrétiennes de la Macédoine est confirmée graduellement après 1904, grâce à l'intervention de l'Europe, et annonce le futur 
dépècement de la région en entités homogènes du point de vue national et le passage de la lutte politique entre les " partis » respectifs à la lutte armée livrée par des bandes nationalistes locales organisées, entretenues et encadrées par l'extérieur ${ }^{8}$. Dans cette «lutte macédonienne» qui combina propagande armée et terreur appliquées aux populations « ambiguës » ou " ennemies ", avec la reconstitution des réseaux partisans en forme politico-militaire, la part du lion appartenait sans doute aux partis grec, bulgare et serbe. On a cependant vu progressivement émerger aussi des bandes armées d'obédience roumaine (avancées par une certaine "Organisation intérieure des Valaques Macédo-Épirotes ", copiée sur le modèle de l'ORIM) ${ }^{9}$, albanaise ${ }^{10}$ et même turco-musulmane - ces derniers d'une nature plus complexe, bien sûr, ressemblant souvent à des escadrons de la mort au service de l'ordre établi ou des propriétaires terriens locaux ${ }^{11}$. Quatre ans après le début de la lutte armée, en printemps 1908, on pouvait cependant discerner les marques d'une évolution sous-jacente en sens inverse et d'une crise profonde des partis et appareils nationaux : saignées, terrorisées, poussées à l'émigration vers les centres urbains ou l'étranger, les populations rurales dans leur majorité absolue ne rêvaient que du rétablissement de la paix ; décimés par la guerre fratricide, les cadres opérationnels des partis rivaux sentaient la futilité de l'entreprise armée. La prépondérance des «hommes d'armes » à l'intérieur de chaque parti national a bouleversé les hiérarchies traditionnelles des communautés en cause ; le terrorisme aveugle des "volontaires» venus d'ailleurs, pour encadrer les bandes locales ou même (surtout dans le cas grec) se substituer les forces locales jugées trop faibles, ou peu promptes à s'entretuer avec leurs voisins, a créé des sentiments forts de

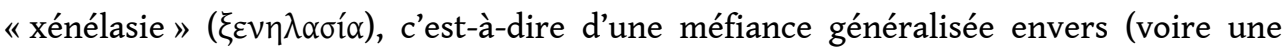
tendance à se débarrasser de) tout émissaire, armé ou pas, des centres nationaux rivaux $^{12}$. L'avènement de la révolution jeune-turque en juillet 1908, et l'annonce des élections parlementaires pour l'automne de cette même année, ont donc servi comme catalyseur pour réorienter les appareils partisans vers une forme d'agitation plus propice aux vœux et besoins de leur clientèle, assurée ou prospective.

7 Canaliser la rivalité nationale dans le cadre d'une confrontation plutôt électorale signifie aussi transformer une nouvelle fois le rapport de forces intérieures dans chaque parti national, écarter les cadres intermédiaires émergés pendant la guerre des bandes et leur substituer des médiateurs pouvant opérer sous les nouvelles conditions ; ces derniers étaient d'habitude issus des couches plus aisées, souvent liés aux familles locales puissantes des marchands urbains ou des tchorbaci villageois. On a donc procédé à une confirmation des hiérarchies sociales et des rapports de classes traditionnelles: l'appareil central des partis et des organisations nationales légales constituées officiellement après le Hürriyet (Union des Clubs Constitutionnels Bulgares, Syllogues Politiques Constitutionnels grecs, Ligues Constitutionnelles Serbes), et surtout les listes des candidats avancées par les appareils pour les postes de député, étaient, dans leur majorité absolue, remplies par des avocats, des juges, des médecins, des employés des consulats ou des Ministères des Affaires étrangères des «centres nationaux", ou au moins des cadres supérieurs des appareils ethno-nationaux du Patriarcat de Constantinople et de l'Exarchat Bulgare. Les "petits profs » (ДАСКАЛЧЕТА) dominant les rangs des factions de l'ORIM, et surtout les « rois de la montagne » enrôlés par toutes les propagandes armées n'étaient désormais plus à la mode. 


\section{La loi électorale}

8 La loi électorale est toujours un facteur déterminant pour dénouer une lutte de ce genre. Aux élections de 1908, on a appliqué une loi votée trois décennies plus tôt par le Parlement de 1876, prévoyant un vote à deux tours ${ }^{13}$ :

- le premier tour qui se faisait au suffrage presque universel (de la population mâle, bien sûr) pour désigner les électeurs de second degré, qui allaient ensuite élire les députés. La loi prévoyait l'élection d'un électeur pour 500 habitants ayant le droit de vote (c'est-à-dire, des habitants «de longtemps » dans l'endroit, de sexe masculin, qui avaient 25 ans au moins et payaient un impôt quelconque); en réalité pourtant, la taille du corps électoral suffisant pour élire un électeur pouvait varier entre 250 et 750 habitants ayant le droit de vote.

- Le second tour prévoyait l'élection des députés par les électeurs du second degré. La circonscription électorale de base était le sandjak, le nombre des députés déterminé par la taille de la population mâle : un député pour 25.000 à 75.000 habitants, deux députés pour une population entre 75.000 et 125.000 , trois députés pour 125.000 à 175.000 , etc. En Macédoine, la population de chaque sandjak était évaluée d'après les résultats du recensement récent de 1905 (dit "de Hilmi Pasha»), mis à jour par les bureaux de population officiels sur la base de l'évolution démographique du pays, mais aussi sur la base des considérations électoralistes du Comité Union et Progrès: ces considérations permettent, par exemple, de constater l'augmentation soudaine et inattendue de la population officielle du sandjak de Drama de 73.000 habitants mâles à 81.850, augmentation qui a permis l'élection des deux députés - au lieu d'un seul - dans cette circonscription électorale de majorité musulmane éclatante.

9 Dans les huit sandjaks composant (dans leur totalité ou en partie) la Macédoine, on a élu vingt-six députés au total : six dans le sandjak de Salonique, quatre dans celui de Serrès, deux à Drama, quatre à Monastir, deux à Korçe, deux à Servia (Serfidje), deux à Debar et quatre à Skopje. Leur répartition entre les différentes " nationalités » a été l'objet d'une série des négociations entre le Comité Union et Progrès (CUP) et les groupes dirigeants des communautés non musulmanes, au niveau central aussi bien qu'à l'échelle régionale. Pour les mouvements et partis nationaux, le plus important, c'était de remporter la majorité dans les régions convoitées : une majorité, au moins relative, surtout face aux mouvements et partis chrétiens rivaux, la population musulmane étant encore considérée comme une entité n'ayant pas des droits «historiques" sur l'avenir du pays. Selon la logique dominante, pendant les crises consécutives de la Question de l'Orient, la corrélation sanctionnée par les urnes servirait moins au futur Parlement ottoman, que comme preuve de "vitalité» nationale aux yeux des Puissances impérialistes du Concert Européen qui, un jour, iraient sans doute surveiller le dépècement prochain de l'Empire ottoman.

Contradiction frappante : au moment où l'on imagine se substituer à l'Empire ottoman dans une partie de ses domaines macédoniens, on prend conscience de sa faiblesse numérique dans la plupart des régions convoitées, et donc de la nécessité d'être appuyé par le Comité Jeune-Turc pour pouvoir faire face aux partis chrétiens rivaux. L'absence d'un quota réservé aux députés non musulmans entraînait le risque de voir les populations chrétiennes - émiettées par l'émergence des partis nationaux- totalement submergées par le vote musulman unifié. Un scénario alternatif, qui donnait, lui aussi des cauchemars aux états-majors des partis nationaux chrétiens, était celui d'une alliance privilégiée entre les Jeunes-Turcs et l'adversaire chrétien: détenteur du 
pouvoir politique, le CUP pouvait facilement instrumentaliser la querelle entre chrétiens pour dominer complètement le terrain, s'érigeant en arbitre de la corrélation des forces régionales ${ }^{14}$.

\section{Pluralisme à la « Jeune-Turc »}

11 Pour le Comité Jeune-Turc, le pluralisme parlementaire idéal devait comprendre une représentation indicative des divers partis nationaux "sur un pied d'égalité », sans aucune relation avec la force numérique des communautés en cause. En plus, on peut clairement discerner une tendance du CUP à favoriser des groupes chrétiens minoritaires dans chaque région, au détriment du Parti national le plus fort sur place et, par conséquent, jugé plus "dangereux» à long terme. Cette promotion des minorités utilisables n'était qu'un signe de continuité avec la politique traditionnelle des autorités ottomanes face à la question macédonienne ${ }^{15}$; elle finira néanmoins par créer pas mal de problèmes dans les relations du Comité avec les mouvements nationaux qui se sentaient trompés par les autorités jeunes-turques. La solution alternative d'une alliance entre les partis chrétiens les plus forts, grec et bulgare, si elle a été formellement envisagée par certains consuls de Grèce et de Bulgarie, était néanmoins encore impensable, étant donné les déchirements réciproques trop récents entre ces mêmes entités collectives ${ }^{16}$.

12 L'aboutissement des négociations contradictoires entre le CUP et les partis nationaux a été la constitution des listes des candidats « mixtes ", soutenues officiellement par les Jeunes-Turcs, et contenant un ou plusieurs délégués de chaque « nationalité » jugée par eux digne d'être représentée au Parlement. Le nombre final des représentants de chaque communauté non musulmane était déterminé, non seulement par la corrélation des forces au niveau local, mais aussi par la conjoncture au niveau international : la proclamation de l'indépendance formelle de la Principauté Bulgare, faite le 22 septembre au milieu de la campagne électorale, a ainsi considérablement contribué à tourner le CUP contre le parti national bulgare représenté par l'Union des Clubs Constitutionnels Bulgares (СОЮЗ НА БЪЛГАРСКИТЕ КОНСТИТУЦИОННИ КЛУБОВЕ, СБКК) récemment créés, en faveur des partis nationaux serbe et grec et du mouvement macédonien autonomiste et socialisant, regroupé autour la personnalité du voïvode Yane Sandanski ${ }^{17}$.

13 À un autre niveau, des rivalités absolument personnelles entre candidats présomptifs à l'investiture de leur parti ont parfois conduit ces derniers à dénoncer aux comités locaux du CUP les liens de leurs rivaux avec l'appareil étatique des pays voisins : ainsi le candidat choisi par le consulat grec (et, ensuite, par la communauté patriarchiste grécisante) de Monastir, un certain Gogos, qui servait comme dragoman à ce même consulat, a-t-il été finalement remplacé, sur l'insistance des Jeunes-Turcs, par l'avocat Traian Nale (ou Nallis), Macédonien slavophone patriarchiste originaire de la région de Morihovo ${ }^{18}$. À Salonique, une tentative semblable, de la part des amis de l'avocat Adamidis, a presque détruit la candidature de Georgios Honaios, dragoman lui aussi du consulat grec de la ville ${ }^{19}$. Beaucoup plus sérieuse a été la révélation faite, juste avant les élections, que le président des Clubs Bulgares, Toma Karaoïvov, candidat du parti bulgare au sandjak de Skopje, était au service du Ministère des Affaires étrangères de Sofia - dénonciation attribuée par le consulat bulgare à un autre personnage local, le publiciste Matej Gerov, qui voulait être désigné candidat à la place du président, mais 
qui a été obligé de présenter sa candidature dans le sandjak voisin de Prizren, où les chances du parti bulgare étaient nulles-. Malgré les déclarations publiques et un peu théâtrales de Karaoïvov en faveur de sa "vraie patrie ottomane » et de son intégrité territoriale, malgré même l'acceptation solennelle de sa part de la prépondérance des musulmans sur les autres éléments de l'Empire, les Jeunes-Turcs ont carrément refusé de soutenir sa candidature et ils ont décidé de faire élire un autre membre du Club bulgare - Todor Pavlov - à sa place ${ }^{20}$.

Les élections ont abouti à la victoire triomphale des listes soutenues par le CUP, exception faite de Servia, où le parti grec l'a emporté en obtenant les deux postes de députés du sandjak après une collision frontale avec la liste locale des Jeunes-Turcs ${ }^{21}$. Dans les sandjaks de Drama et de Debar, à forte majorité musulmane, on n'a élu que des députés musulmans ${ }^{22}$. Dans les autres cinq sandjaks pourtant, le déroulement du suffrage a été plein de suspens, et le résultat parfois imprévu. Un système d'alliances variables et les violations des accords par l'un ou l'autre parti ont beaucoup contribué à cette imprévisibilité. De surcroît, les autorités jeunes-turques semblent avoir décidé de ne rien laisser au hasard, utilisant une série des trucages électoraux pour s'assurer le soutien du plus grand nombre possible d'électeurs de second degré et transformer ainsi leurs « alliés » en vrais otages.

Parmi les accusations formulées contre le CUP, en ce qui concerne le trucage des élections de 1908, la plus substantielle semble être un dessin des circonscriptions électorales de premier degré et des bureaux de vote tels que des villages ou quartiers à majorité musulmane puissent élire un électeur pour 250 ou 300 habitants ayant le droit de vote, tandis que pour les villages à majorité chrétienne, la proportion correspondante était de un électeur pour 700 à 750 votants $^{23}$. On a aussi toute raison de suspecter la découverte annoncée au dernier moment avant le scrutin, selon laquelle la population mâle du sandjak de Drama, - que le recensement récent de 1905 avait estimé à environ 73.000 hommes - était, en réalité, composée de 81.850 hommes, une différence cruciale, qui a permis d'y élire deux députés (musulmans) au lieu d'un seul ${ }^{24}$. D'autres irrégularités ont été aussi dénoncées: manque de vérification des listes électorales au niveau du village ou du quartier comme il était prévu par la loi, exclusion arbitraire des votants qui satisfaisaient pourtant à toutes les conditions pour avoir le droit de vote, trucage pur et simple du contenu des urnes, comme dans le cas de la ville de Voden (aujourd'hui Edessa), où les bulletins de votes "grecs » et «bulgares » ont été, en force, remplacés par des pièces de papier contenant des noms des électeurs de second degré musulmans ${ }^{25}$.

\section{La bataille des urnes}

Dans le sandjak de Salonique, la liste officielle des candidats jeune-turcs, annoncée le 21 septembre, prévoyait l'élection de deux députés musulmans, de deux «Grecs » (c-à-d. patriarchistes), d'un «Bulgare» (c-à-d. exarchiste) et d'un Israélite. Les deux musulmans étaient les beys Rahmi et Djavit, les «Grecs ", l'avocat Georgios Artas (élu de la communauté patriarchiste de la ville) et l'ex-dragoman Honaios, le "Bulgare ", l'avocat Tilkov proposé par ськк, et l'Israélite, l'avocat Emmanuel Karasso, membre du CUP ${ }^{26}$. Le premier tour des élections dans le sandjak a eu lieu pendant le mois d'octobre et aboutit à l'élection de 120 électeurs de second degré musulmans, 95 "Grecs », 39 «Bulgares », 2 «Valaques » (c'est à dire adhérents au parti roumain, le parti «Grec » 
ayant aussi «ses» Valaques) et 17 Israélites ${ }^{27}$. C'était une victoire claire du Parti grec sur son adversaire bulgare, victoire due, selon le consul grec de la ville, à trois facteurs principaux : (a) l'existence de trois kazas plus au sud (Katerini, Veroia et Kassandra) où l'élément «purement grec» (c'est-à-dire chrétien grécophone) était dominant, assurant une majorité écrasante de 50 électeurs sur 60 au parti grec, (b) la coopération entre patriarchistes et musulmans dans les kazas de Gevgelja, Doiran et d'ailleurs, qui a enterré les chances du parti bulgare local, et (c) le déchirement entre les partis bulgare et macédonien pour l'hégémonie au sein de la population exarchiste et (plus généralement) slavophone, qui les a empêché de faire front unique contre les autres groupes ethniques et / ou nationaux ${ }^{28}$. Au deuxième tour, tous les candidats proposés par les Jeunes-Turcs ont été élus, sauf un: l'avocat Tilkov de l'Union des Clubs Constitutionnels Bulgares, qui a été défait par le professeur socialiste Dimitar Vlahov, candidat du parti bulgaro-macédonien de Yane Sandanski, soutenu au dernier moment par l'appareil jeune-turc en réaction à la déclaration de l'indépendance de la Bulgarie $^{29}$; en ce qui concerne le vote «bulgare ", Vlakhov raconte dans ses mémoires qu'il a gagné les électeurs exarchistes des kazas de Strumitsa et de Tikfeš, tandis que ses compatriotes de Kilkis ont préféré soutenir son rival du Club nationaliste ${ }^{30}$. Néanmoins, le CUP n'a pas été le seul acteur à briser l'accord préélectoral : dans leur grande majorité, les électeurs "grecs" se sont abstenus de voter pour un candidat «bulgare " quel qu'il soit, en donnant leur vote à un troisième candidat grec indépendant de la région de Chalcidique, G. Samaras, qui a failli être élu, lui aussi. Furieux de cette "violation des règles du jeu », et désirant rappeler le parti grec à (leur) ordre, les Jeunes-Turcs ont menacé de ne pas faire élire le candidat grec du sandjak de Serrès.

17 L'éclatement du parti «bulgare » a été beaucoup plus sévère - et crucial - dans le sandjak voisin de Serrès, où la prépondérance numérique des chrétiens slavophones était officiellement acceptée, même par les autorités ottomanes: tandis que les résultats officiels du recensement de 1905 donnaient un total de 182.689 habitants permanents mâles dans tout le sandjak (dont 73.899 musulmans, 66.872 exarchistes et 39.585 patriarchistes) ${ }^{31}$, sa révision publiée par le Comité Jeune-Turc en septembre 1908 projetait une nette majorité nette de 186.695 «Bulgares» (des deux sexes) (ou 47,5\%) contre 139.710 musulmans $(35,5 \%)$ et 37.560 « Grecs » seulement $(9,5 \%)^{32}$. Il ne faut pas donc s'étonner du fait que l'appareil bulgare n'ait fait que s'entre-déchirer, le consulat de la ville cherchant surtout à combattre l'influence du "parti macédonien " de Sandanski parmi la population slavophone; de plus, l'organisation politique de ce dernier n'accordait pas d'attention sérieuse à la lutte électorale, tout en bloquant la construction des Clubs Constitutionnels rivaux dans la zone qu'elle contrôlait. Le poids numérique des "Bulgares" a pourtant provoqué une alliance entre leurs rivaux «Grecs » et musulmans : dès le 27 juillet, un accord entre le Comité Jeune-Turc local et le consul de la Grèce était déjà conclu, selon lequel le parti grec devait obtenir un des quatre sièges du sandjak. Effrayés par cette coopération qui pouvait priver le parti bulgare de toute représentation parlementaire, la ськК de Salonique a conclu un accord semblable avec le CUP, prévoyant l'élection d'un seul député bulgare ${ }^{33}$. La déclaration de l'indépendance bulgare a conduit à de nouveaux changements dans la politique des autorités ottomanes. Fin septembre, on a ainsi sondé le consulat grec sur la possibilité de remplacer le seul député "bulgare» prévu par un "roumain», totalement fidèle au statu quo $0^{34}$. Enfin, le 9 octobre Yane Sandanski - qui jusqu'alors flirtait avec l'idée d'une dénonciation complète du scrutin - a annoncé qu'il allait aussi 
y participer, en compagnie de Hristo Daltchev, un avocat de Salonique bien connu dans la région comme défenseur des prisonniers politiques pendant l'ancien régime; les «clubistes» ont aussi contacté Daltchev qui avait pourtant rejeté poliment leur offre avant de s'allier avec leur ennemi politique ${ }^{35}$. Le Comité Jeune-Turc a annoncé que, de son côté, il donnait son aval aux trois candidats, en morcelant ainsi pratiquement les effets bénéfiques du vote musulman pour les partis exarchistes ${ }^{36}$.

Le résultat de ce jeu d'alliances multiples et de « vote croisé » fut catastrophique pour la représentation slave. Le premier tour a donné la majorité absolue aux électeurs musulmans (92 sur 176 ou 52,3\%), les électeurs «bulgares » réduits à 50 seulement contre 34 «Grecs $»^{37}$. Au second tour, les deux candidats musulmans du bulletin jeuneturc ont été triomphalement élus par les voix des 176 électeurs; le candidat grec Dimitrios Digas, avocat lui aussi à Salonique, a été élu avec 100 voix ; parmi les trois candidats « bulgares", c'est l'avocat Daltchev qui l'a finalement emporté avec 84 voix, contre 50 pour Sandanski et 46 pour le candidat des Clubs nationalistes, Todor Paskaliev, secrétaire de la métropole exarchiste de Skopje, mais originaire du sandjak de Serrès ; les candidats indépendants Akil bey (musulman) et Nicolae Batzaria (valaque du parti roumain) ont obtenu 24 et 18 voix respectivement ${ }^{38}$. La répartition du vote de chaque groupe ethnique au niveau du kaza révèle l'existence d'alliances locales pas (complètement) adaptées aux plans élaborés par les appareils centraux: d'après le consul grec,

"les Bulgares ont voté pour des Grecs dans le kaza de Petritch et les Grecs ont voté pour des candidats bulgares à Petritch et à Melnik", tandis que "les Ottomans [c'est-à-dire les musulmans] n'ont pas voté pour notre candidat dans les kazas de Serres, de Razlog et de Gorna Djoumaya $»^{39}$.

19 Selon une enquête spéciale post-électorale du consulat bulgare, le vote exarchiste a été disséminé de façon variée d'un kaza à l'autre. Tous les électeurs de Gorna Djoumaya, la majorité écrasante (cinq sur six) de ceux de Serrès et un sur six dans celui de Demir Hissar ont voté comme "centralistes convaincus» - partisans fermes du parti macédonien - pour le tandem Sandanski-Daltchev; les électeurs bulgares de Petritch ont voté seulement pour Daltchev (et le candidat grec Digas), en rejetant les deux versions de la lutte fratricide ; tous les électeurs de Razlog et cinq sur six à Demir Hissar ont voté pour Daltchev et le «clubiste» Paskalev, tandis que tous ceux de Melnik ont pris une position radicalement anti-Sandanski, en votant seulement pour Paskalev (et Digas); les onze électeurs de Nevrokop ont tous voté pour le candidat nationaliste bulgare, en divisant leur seconde voix entre Sandanski, Daltchev et Digas; les deux électeurs exarchistes du kaza de Zihna ont enfin voté simultanément pour Sandanski et Paskalev ${ }^{40}$.

La défaite humiliante de Sandanski, chef principal du parti macédonien issu de l'aile gauche du mouvement révolutionnaire bulgare, était sans doute le plus signifiant de ces résultats. Ses biographes, qui ont une tendance naturelle à le glorifier, ainsi que ses partisans dans l'historiographie macédonienne, s'avèrent, pour la plupart, réticents à se prononcer sur ce point, le plus souvent en évitant toute référence à sa mésaventure électorale ${ }^{41}$. L'explication la plus élaborée est formulée par Mercia MacDermott: «le suffrage limité, le système à deux tours et les irrégularités étendues pendant le scrutin ", écritelle, "ont tous contribué à minimiser l'importance des élections comme expression d'une opinion publique authentique $»^{42}$. En fait, Sandanski et ses camarades ont beaucoup hésité avant de se lancer dans la bataille des urnes; mais exactement le même comportement fut aussi observé parmi leurs rivaux du parti bulgare nationaliste, exaspérant les 
représentants diplomatiques de Sofia dans la région ${ }^{43}$. Dans leurs rapports, les diplomates bulgares ne cachent leur animosité, sinon leur haine, contre le chef du parti macédonien, contre lequel ils avaient renu l'ordre de mobiliser toute leur énergie ; en dressant le bilan des élections, ils ont ainsi une tendance explicable à surestimer la contribution musulmane dans les votes qu'il a requs, ou même à le considérer comme "un candidat des Turcs » ${ }^{44}$. Plus fiables sont les informations qu'on puise dans des sources extérieures au camp bulgaro-macédonien : selon le consul anglais de Salonique, par exemple, le CUP avait eu une attitude très négative face à sa candidature, et il a essayé de la bloquer en invoquant son ignorance de la langue turque ; le consul grec à Serrès rapporte de son côté que, après accord avec le parti grec, le Comité Jeune-Turc a ordonné qu'on ne vote plus pour Sandanski ${ }^{45}$. En tout cas, Sandanski - mis hors combat par l'attentat contre lui le 24 septembre à Salonique - devait surmonter tout le dispositif de l'État bulgare et même de quelques consuls européens pro-bulgares qui "s'agitaient terriblement contre lui ", sur un terrain dont il n'était d'ailleurs pas le meilleur connaisseur ${ }^{46}$.

21 Le même scénario s'est plus ou moins reproduit dans le sandjak de Monastir, à la différence que le camp exarchiste y était beaucoup plus solide. La composition ethnonationale de la population ressemblait aussi à celle de Serrès : selon l'estimation des autorités, $47 \%$ des 171.364 habitants étaient des «Bulgares ", $30 \%$ des musulmans, $14,2 \%$ des «Grecs", $6,8 \%$ des «Serbes », $1,3 \%$ des israélites et $1,4 \%$ des «Roumains ${ }^{47}$. Pour les partis grec et serbe, la seule voie possible était donc la coopération avec les Jeunes-Turcs, pour assurer une certaine représentation parlementaire ${ }^{48}$. L'appareil bulgare, au contraire, a commencé par rêver acquérir la totalité des quatre sièges du sandjak; vers la fin de septembre, le consul de Sofia à Monastir considérait encore que le parti bulgare pouvait élire au moins deux députés ${ }^{49}$. Érigé en arbitre, le Comité Jeune-Turc local a enfin changé plusieurs fois d'opinion pendant cet automne: en commençant par suggérer deux députés "bulgares", un «turc» et un "grec », il est passé ensuite à deux "Turcs», un «Bulgare » et un «Grec » pour arriver à la proposition finale d'une représentation équitable des quatre nationalités différentes, en ajoutant un député "serbe», le docteur Yannaki Dimitrievitch $^{50}$. Le parti bulgare a de côté examiné plusieurs possibilités; une délégation de l'Organisation révolutionnaire intérieure macédonienne a même proposé au consulat grec une alliance "gréco-bulgare " qui aurait fait élire deux députés "bulgares» et deux "Grecs», dont le second aurait dû être indiqué par l'organisation ${ }^{51}$. Finalement, on a cependant formé un bulletin composé de quatre candidats uniquement exarchistes: le procureur du Cour d'Appel Pantcho Dorev (supporté aussi par les Jeunes-Turcs), le juge Anton Dimitrov (ancien cofondateur de l'ORIM), le docteur Georgi Nikolov et l'ancien secrétaire de la communauté exarchiste de Salonique - né a Prilep - Anastas Hristov ${ }^{52}$.

Les résultats du scrutin furent, une fois encore, destructeurs pour le parti bulgare. Grâce à l'action coordonnée des autorités ottomanes avec les partis grec et serbe, le tracé très compliqué des circonscriptions électorales, l'exclusion des communautés exarchistes sous prétexte qu'elles n'avaient pas payé un impôt et la réaction spontanée d'une partie de la base sociale du parti (qui a décidé de s'abstenir aux élections en guise de protestation), l'alliance anti-exarchiste a encore une fois prévalu: d'après les résultats légèrement différents rapportés par les consuls de Grèce et de Bulgarie, on a élu 62-64 électeurs musulmans, 52-55 «Bulgares », 15-20 «Grecs », sept «Serbes » et 
deux Israélites ${ }^{53}$. Selon l'exposé détaillé post-électoral de ськк, le kaza de Florina constitua l'exemple le plus frappant de la politique jeune-turque de tracer les circonscriptions électorales d'une manière propre à renverser totalement le rapport des forces réel sur le terrain. Voici par exemple la répartition des bureaux de vote dans la ville même de Florina, où le parti bulgare n'a pas pu élire un seul électeur sur neuf, en tenant compte l'appartenance ethno-religieuse des votants ${ }^{54}$ :

Répartition des bureaux de vote dans la ville de Florina

\begin{tabular}{|l|l|l|l|l|}
\hline \multirow{2}{*}{ Bureau de Vote } & \multicolumn{4}{l|}{ Votants } \\
\cline { 2 - 5 } & Total & Musulmans & Patriarchistes & Exarchistes \\
\hline $1^{\mathrm{er}}$ & 2.874 & 1.362 & 419 & 1.093 \\
\hline $2^{\mathrm{e}}$ & 1.089 & 103 & 593 & 393 \\
\hline $3^{\mathrm{e}}$ & 993 & 599 & 67 & 327 \\
\hline Total & 4956 & 2064 & 1079 & 1810 \\
\hline
\end{tabular}

Ce trucage, ainsi que les réactions infructueuses du parti bulgare, sont dans une grande mesure corroborés par les rapports du consul grec de Monastir relatifs aux élections dans le kaza de Florina ${ }^{55}$. En plus, il semble qu'un procédé identique a été mis en pratique dans d'autres centres urbains du même sandjak ${ }^{56}$. L'appareil bulgare a bien sûr protesté, mais pour être réduit au silence par la réponse définitive du Comité JeuneTurc: la répartition discutée avait été entérinée par les signatures des deux représentants de la communauté exarchiste au comité électoral du sandjak $!^{57}$ Sans le nier, ces derniers ont tenté de s'excuser en parlant d'un «malentendu " qu'ils ont ensuite essayé en vain de repérer. Naturellement, l'appareil bulgare décida de ne plus toucher au sujet ${ }^{58}$. Au second tour des élections, les quatre candidats du bulletin officiel l'ont naturellement remporté, avec Pantcho Dorev clairement en tête devant les autres ${ }^{59}$.

Dans le sandjak voisin de Korçe, habité surtout par des populations albanophones et dont une partie appartenait à la Macédoine (kaza de Kastoria, nahiye de Biglishta), la même politique fut appliquée par les autorités jeunes-turques contre le parti grec puissant. D'après le recensement de 1905, les 99.348 habitants du sandjak étaient à $46 \%$ "Grecs » (orthodoxes patriarchistes), à 44,6\% musulmans, à $7 \%$ «Bulgares » (orthodoxes exarchistes) et à $2,1 \%$ «Roumains » (valaques orthodoxes appartenant au parti roumain $)^{60}$. Le CUP avait décidé que le Parti roumain devait aussi être représenté dans le futur Parlement; ses propositions sur ce sujet étant rejetées par les autres partis nationaux (surtout le parti grec) dans les sandjaks de Serrès et de Monastir, on a finalement décidé de faire élire le député « roumain »| par la population du sandjak de Korçe, même si le parti roumain local ne contrôlait qu'un seul village ; un tel choix était d'ailleurs favorisé par la politique de "divide et impera» des autorités ottomanes, dirigée chaque fois contre le parti le plus fort parmi les populations chrétiennes locales $^{61}$. L'appareil grec a, à son tour, refusé d'obéir et il a essayé de faire front avec les autres éléments locaux qui se sentaient aussi écartés par le choix des autorités - dans 
ce cas-là, avec les Turcs « caşabalis » (c'est-à-dire habitants du centre de la capitale du sandjak), marginalisés face au développement du mouvement national albanais. Les élections ont été par conséquent transformées en confrontation de deux blocs d'alliances croisées traversant les lignes de séparation religieuses : d'une part les partis albanais, roumain et bulgare, appuyés par l'appareil jeune-turc, d'autre part les partis grec et turc ${ }^{62}$. Grâce aux interventions ouvertes des institutions étatiques en faveur du premier bloc et la répartition des circonscriptions électorales « à la Monastir » (dans la même ville de Korçe, on n'a pas élu un seul électeur du parti grec!), la déroute de l'alliance gréco-turque fut complète. Le premier tour a abouti à l'élection de 45 électeurs musulmans sur 71, le parti grec n'obtenant que 19 élus contre 6 au parti bulgare (tous au kaza de Kastoria) et un seul du parti roumain. Comme c'était prévu, au second tour, on a élu comme députés Sahin Bey de Kolonia, chef du réseau albaniste et ancien éditeur du journal « Drita » à Sofia, et le docteur « roumaniste » Miše ${ }^{63}$.

Dans le sandjak de Skopje, le parti serbe minoritaire (7\% de la population selon le recensement de 1905 et les estimations de la métropole exarchiste) était érigé de facto en arbitre entre le parti bulgare majoritaire ( $49 \%$ de la population) et la communauté musulmane, en majorité albanaise $(44 \%)^{64}$. Dès le début, le consul bulgare de la ville s'était prononcé pour une alliance des deux communautés chrétiennes prévoyant un soutien réciproque dans les sandjaks de Skopje (des «Bulgares » par les "Serbes ») et de Tetovo, appartenant au vilayet de Prizren (vice versa), tout en reconnaissant les difficultés d'une telle évolution: après la confrontation sanglante des années précédentes entre bandes armées des deux partis, il explique, celui qui osera proposer le premier une coopération électorale serbo-bulgare "va être battu» par ses compatriotes et compagnons ${ }^{65}$. Finalement, une alliance a été formée au sens inverse, contre le parti bulgare, entre Serbes et Jeunes-Turcs. Les résultats du premier tour du scrutin ont ainsi abouti à une nette majorité musulmane: 85 électeurs contre 78 «Bulgares» et 11 «Serbes» selon le rapport détaillé du consulat bulgare, 95 musulmans contre 56 " Bulgares » et 18 "Serbes » selon celui, beaucoup plus sommaire, $\mathrm{du}$ consulat $\operatorname{grec}^{66}$. Au deuxième tour, on a élu deux députés musulmans (le très riche albanais Nedjip bey, à la tête d'un cartel monopoliste du commerce du bois dans tout le vilayet de Kossovo, et Sait Hodja, un maitre d'école vétéran du mouvement jeune-turc), un député «bulgare » (Todor Pavlov, professeur à l'école bulgare et la Faculté du Droit de Constantinople) et un député "serbe" (le pharmacien Alexandar Parlitch, ancien cadre du parti bulgare au kaza de Tetovo qui a passé ensuite au service du parti serbe ${ }^{67}$. Les effets de la coopération serbo-ottomane mis à part, la défaite du parti bulgare a été déterminée aussi par des facteurs de dissidence interne. Dans la ville de Kratovo, par exemple, l'inimitié régnant parmi les notables exarchistes à cause des "petites choses personnelles insignifiantes " et la mémoire d'une confrontation récente entre citadins et villageois, provoquée par l'exécution récente d'un chef révolutionnaire local, ont entravé l'élection des électeurs chrétiens. Dans le kaza de Pehčevo, contrôlé par le parti macédonien de Hristo Tchernopeev, il n'y avait ni Clubs nationalistes, ni intelligence locale; le consul de Skopje a donc été obligé de négocier avec les représentants des classes populaires, les chefs d'armes traditionnelles de la région montagneuse de Males et les « gens des esnafs » de la ville, pour assurer l'élection d'au moins quatre électeurs «bulgares ${ }^{68}$. La répartition des circonscriptions électorales était, par contre, favorable ici au Parti bulgare plutôt qu'au parti serbe ${ }^{69}$. Quant au parti grec minuscule du sandjak, il a suivi une politique d'alliance avec le parti serbe ou avec les notables ottomans locaux, selon les circonstances; sa grande victoire fut, néanmoins, le regroupement - à 
l'occasion du scrutin - d'une faction de la base sociale du parti roumain parmi les Valaques de Skopje ${ }^{70}$.

\section{Face à l'Histoire}

Le bilan des premières élections parlementaires en Macédoine ottomane reste donc assez ambigu. Leur image est radicalement différente dans la bibliographie balkanique et dans celle de la Turquie ou des pays occidentaux. Les historiographies balkaniques, plus ou moins nationalistes, n'y voient qu'une série d'irrégularités et de trucages subis par leur parti national respectif - en oubliant, d'habitude, les cas inverses de partialité jeune-turque en leur faveur. L'historiographie turque, même la moins bien disposée envers le régime du CUP, considère les élections de 1908 comme les seules dignes de ce nom pendant toute la période ${ }^{71}$. Les historiens occidentaux spécialisés sont souvent du même avis, ou reproduisent simplement le point de vue des consuls européens de l'époque, plus ou moins satisfaits du comportement électoral des Jeunes-Turcs. En fait, les représentants diplomatiques des Puissances ne s'intéressaient pas du tout aux performances démocratiques du nouveau régime, mais plutôt au maintien d'un équilibre pour ne pas déstabiliser la paix européenne et mondiale: consuls et ambassadeurs d'Angleterre et d'Autriche-Hongrie ont ainsi rapporté avec satisfaction que, après les irrégularités de toutes sortes observées au premier tour, les Jeunes-Turcs n'ont pas exploité leur majorité pour étouffer complètement les partis nationaux chrétiens, mais leur ont accordé un nombre des sièges parlementaires à peu près correspondant à la taille numérique de leur base sociale ${ }^{72}$. Il ne faut pas pourtant oublier que les puissances en cause ressentaient en même temps une inquiétude réelle face à l'éventualité d'un développement vraiment démocratique de la Nouvelle Turquie, qui aurait servi de "mauvais exemple» aux peuples coloniaux administrés par eux ${ }^{73}$. De son côté, l'ambassadeur de Russie à Constantinople n'a vu dans la "farce » électorale de 1908 que l'incapacité bien enracinée des peuples orientaux à mettre en valeur les libertés et les systèmes d'autogouvernement " occidentaux »; de plus, il était très ennuyé par le fait que la communauté juive de Salonique serait, elle aussi, représentée dans le futur Parlement, sans compter la présence dans les rangs parlementaires d'un «socialiste extrémiste » comme Dimitar Vlahov, un homme «sans aucun sentiment national $»^{74}$.

Il y a, enfin, le cas des élections ottomanes suivantes de 1912, bien connues - dans l'historiographie turque aussi bien qu'occidentale - comme «les élections du gros bâton", qui justifient une certaine indulgence envers le précédent de 1908. Cette dégénérescence crépusculaire, liée à la déception graduelle de la population et de la classe politique face au régime de plus en plus autoritaire du CUP, n'est cependant pas comparable avec la «lune de miel», pleine de promesses et d'espérance, de la révolution jeune-turque. Il semble, néanmoins, que le modèle politique projeté dans ce premier test du nouveau régime était bien indicatif de son évolution postérieure. 


\section{BIBLIOGRAPHIE}

Adanir, Fikret, (1979), Die Makedonische Frage, Ihre Entstehung und Entwicklung bis 1908. Wiesbaden: Frankfurter Historische Abhandlungen, Band 20. Franz Steiner Verlag.

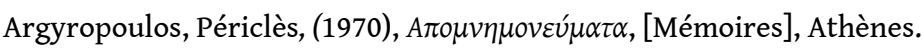

Bérard, Victor, (1900), La Macédoine, Paris.

Clayer, Nathalie, (2007), Aux origines du nationalisme albanais, Paris : Karthala/CERI, coll.

« Recherches internationales».

Cvijic, Jovan, (1917), Questions Balkaniques, Paris-Neuchatel.

Dakin, Douglas, (1966), The Greek Struggle in Macedonia 1897-1913, Salonique: IBS.

Devereux, Robert, (1963), The first Ottoman Constitutional period, a study of the Midhat constitution and parliament. The Johns Hopkins University Studies in Historical and Political Science, Ser. LXXXI, NO. 1. Baltimore: Johns Hopkins Press.

Eliot, Charles, (1908), Turkey in Europe, $2^{\mathrm{e}}$ éd., Londres.

Feroz, Ahmad, (1969), The Young Turks, the Committee of Union and Progress in Turkish politics, 1908-1914, Oxford: Clarendon Press.

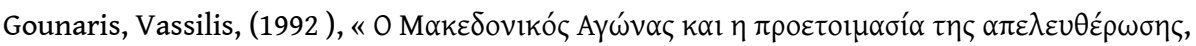
1903-1912 », [La lutte macédonienne et la préparation de la libération 1903-1912] dans

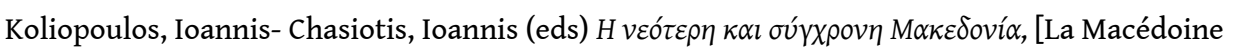
moderne et contemporaine] Athènes-Salonique, vol. I.

Hanioglu, Sükrü, (2008), « The Second Constitutional Period, 1908-1918 », dans Reşat Kasaba (ed.), The Cambridge History of Turkey, vol. 4, Cambridge: Cambridge University press.

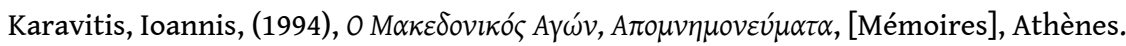

Kayali, Hasan, « Elections and the Electoral Process in the Ottoman Empire, 1876-1919 », International Journal of Middle East Studies, 27 (1995), pp. 266-7.

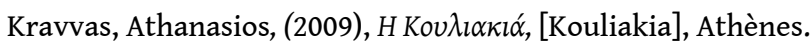

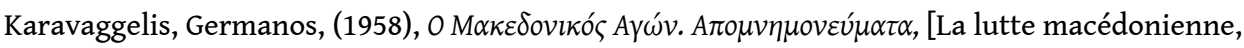
Mémoires], Salonique.

Lowther, Sir Georges, (1928), “Annual Report on Turkey for the Year 1908”, dans G.P. Gooch - H. Temperley, British Documents on the Origins of the War, 1898-1914, vol. V., The Near East, Londres.

MacDermott, Mercia, (1988), For freedom and perfection: the life of Yane Sandansky, Londres: Journeyman.

Makedonski, Stojan, (1974), « La révolution Jeune-Turque et les premières élections parlementaires de 1908 en Macédoine et en Thrace Orientale ", Études Balkaniques, 4.

Marinov, Tchavdar, (2006), « L'impasse du Passé. La Construction de l'identité nationale macédonienne et le conflit politico-historiographique entre la Bulgarie et la Macédoine ", Thèse doctorale à l'EHESS, Paris.

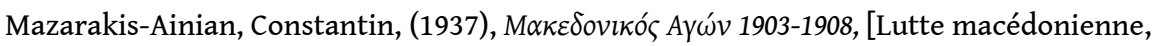
1903-1908], Salonique. 
Reiss, Rudolf, (1915), Rapport sur la situation des bulgarophones et des musulmans dans les nouvelles provinces grecques, s.l.

Sonnischen, Albert, (1909), Confessions of a Macedonian Bandit, N. York 1909.

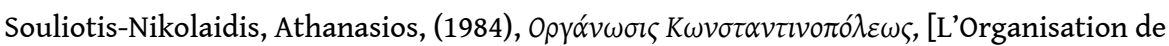
Constantinople], Athènes-Ioannina.

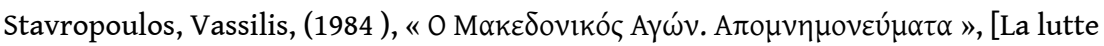

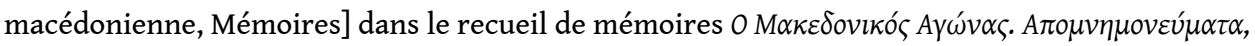
[La lutte macédonienne, Mémoires], Athènes.

The University 'Cyril and Methodius', (1985), Documents on the Struggle of the Macedonian People for Independence and a Nation-State, Skopje.

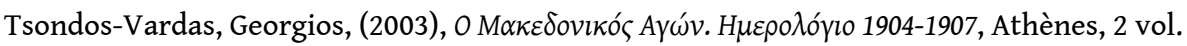

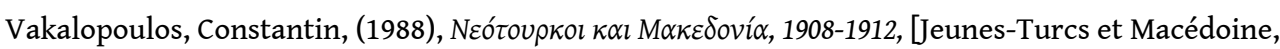
1908-1912] Salonique.

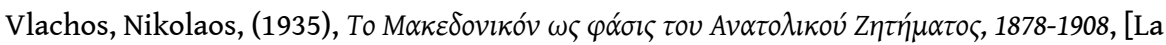
question macédonienne comme phase de la question d'Orient, 1878-1908], Athènes.

ИНСТИТУТ ЗА НАЦИОНАЛНА ИСТОРИјА, ИСТОРИјА НА МАКЕДОНСКИОТ НАРОД, Skopje, 1969, vol. II; [Institut of National History, A history of the Macedonian people, Skopje, 1979; Institut of National History, History of the Macedonian people] Skopje, 2008.

АТАНАС ШОПОВ, (1893), ИЗ ЖИВОТА И ПОЛОЖЕНИЕТО НА БЪЛГАРИТЕ В ВИЛАЕТИТЕ, Plovdiv.

ГЕОРГИ БАЖДАРОВ, (1929), ГОРНО-БРОДИ, Sofia.

ВАСИЛ КЪНЧОВ, (2000), МАКЕДОНИЯ. ПЪТЕПИСИ, Sofia.

ВЕЛИЧКО ГЕОРГИЕВ, СТАЙКО ТРИФОНОВ, (1995), ГРЪЦКАТА И СРЪБСКАТА ПРОПАГАНДИ В МАКЕДОНИЯ, Sofia.

ХРИСТО СИЛЯНОВ, (1943), ОСВОБОДИТЕЛНИТЕ БОРБИ НА МАКЕДОНИЯ, СЛЕД ИЛИНДЕНСКОТО ВъЗСТАНИЕ, Sofia.

АЛЕКСО СТЕФАНОВ, (1931), РЕВОЛЮЦИОННАТА ДЕЙНОСТ В ДЕМИРХИСАР (БИТОЛСКО), Sofia.

ПЕТЪР СВИРАЧЕВ, ДАРИНА ИЛИЕВА, (1992), « ПОВЕРИТЕЛЕН ДОКЛАД ОТ АНДРЕЙ ТОШЕВ ПО

МАКЕДОНСКИЯ ВЪПРОС ОТ 1907 Г. », ИЗВЕСТИЯ НА ДЪРЖАВНИТЕ АРХИВИ, 64.

КИРИЛ ПЪРЛИЧЕВ, (2001), КЮСТЕНДИЛСКИЯТ КОНГРЕС НА ВМРО, 1908 Г., Sofia.

ВЛАДИМИР КАРАМФИЛОВ, (2005), СПОМЕНИ ЗА ПРОСВЕТНОТО ДЕЛО И РЕВОЛЮЦИОННИТЕ БОРБИ В ГР. ПРИЛЕП, Sofia.

СТЕФАН САЛГЪНДЖИЕВ, (1906), ЛИЧНИ ДЕЛА И СПОМЕНИ ПО ВЪЗРАЖДАНЕТО НА СОЛУНСКИТЕ И СЕРСКИ БЪЛГАРИ, Plovdiv.

ГЕОРГИ ТРАЙЧЕВ, (1930), СПОМЕНИ ОТ МОЕТО 40 ГОДИШНО УЧИТЕЛСТВО, Sofia.

ИВАН СНЕГАРОВ, (1937), СОЛУН В БЪЛГАРСКАТА ДУХОВНА КУЛТУРА, Sofia.

ДАНЧО ЗОГРАФСКИ, (1981), АВСТРИСКИ ДОКУМЕНТИ ЗА ИСТОРИЈАТА НА МАКЕДОНСКИОТ НАРОД. ТОМ ІІ (1907-1908), Skopje.

ПАВЕЛ ДЕЛИРАДЕВ, (1946), ЯНЕ САНДАНСКИ, Sofia.

АНГЕЛ ДИНЕВ, (1987), ИЛИНДЕНСКАТА ЕПОПЕјА, Skopjе. 
ИВА БУРИЛКОВА, ЦОчО БИЛЯРСКИ, (2007), « ПРЕДГОВОР », dans le recueil ЯНЕ САНДАНСКИ. СПОМЕНИ, Sofia.

К.А. Kochmanski : СИМОН ДРАКУЛ (ed.), (2006), МАКЕДОНИјА МЕЃУ АВТОНОМИјАТА И ДЕЛЕЖОТ. ЗБОРНИК РУСКА ДИПЛОМАТСКА ДОКУМЕНТАЦИјА -1894-1913, vol. VI, Skopje.

\section{ANNEXES}

Résultats électoraux du $1^{\text {er }}$ tour par sandjaks et kazas

\section{Sandjak de Salonique}

\begin{tabular}{|l|l|l|l|l|l|l|}
\hline \multirow{2}{*}{ KAZA } & \multicolumn{4}{|l}{ Électeurs de $2^{\mathrm{e}}$ degré } & \multicolumn{4}{l|}{} \\
\cline { 2 - 7 } & TOTAL & Musulmans & Grecs & Bulgares & Valaques & Israélites \\
\hline Salonique & 63 & 15 & 24 & 2 & - & 15 \\
\hline Kassandra & 27 & 2 & 25 & - & - & - \\
\hline Langada & 21 & 12 & 9 & - & - & - \\
\hline Veroia & 20 & 3 & 15 & - & 2 & - \\
\hline Vodena & - & - & - & - & - & \\
\hline Karadjova & 13 & 10 & - & 3 & - & - \\
\hline Yenidje & - & - & - & - & - & \\
\hline Katerini & 13 & 3 & 10 & - & - & - \\
\hline Avret Hissar & 14 & 6 & - & 8 & - & - \\
\hline Dö̈ran & 11 & 11 & - & - & - & - \\
\hline Gevgelija & 19 & 11 & 5 & 2 & - & - \\
\hline Strumica & 26 & 12 & 6 & 7 & - & 1 \\
\hline Tikfeš & 13 & - & - & - & - & - \\
\hline TOTAL & 265 & 120 & 95 & 39 & 2 & \\
\hline & & & & & & \\
\hline
\end{tabular}

Sources : IAYE 1908/101, A. Papadiamantopoulos au MAE G. Baltatzis, Salonique

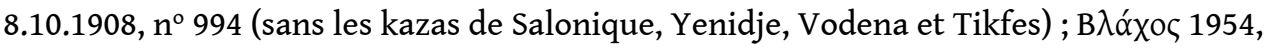

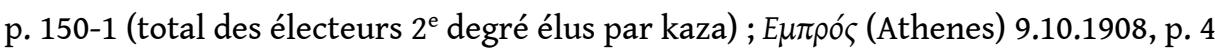
(kaza de Veroia), 15.10.1908, p. 4 (kaza de Salonique, sauf le bureau de vote de Ayia Triada) et 13.10.1908, p. 4 (9 kazas). Cet dernier article donne des résultats légèrement différents pour les kazas de Langada (12 « Turcs »+ 9 « Grecs ») et d'Avret Hissar (11 
«Bulgares »+8 « Turcs »), tandis que ses données pour le kaza de Karadjova sont manifestement incomplètes (deux « Bulgares » + deux « Turcs »). Les sources bulgares à ma disposition donnent des résultats partiels légèrement différents. Dans le kaza de Strumica, par exemple, le rapport de la ськк mentionne 27 électeurs : 12 musulmans, 1 Israelite, 5 « Grecs » et 9 « Bulgares » (ЦДА, Ф.176К, оП.2, А.Е.144, Л.26).

\section{Sandjak de Serrès}

\begin{tabular}{|l|l|l|l|l|}
\hline \multirow{2}{*}{ KAZA } & \multicolumn{4}{|l|}{ Électeurs de 2e degré } \\
\cline { 2 - 5 } & TOTAL & Musulmans & Grecs & Bulgares \\
\hline Serres & 54 & 25 & 23 & 6 \\
\hline Zihna & 17 & 8 & 7 & 2 \\
\hline Demir Hissar & 18 & 10 & 2 & 6 \\
\hline Melnik & 13 & 6 & - & 7 \\
\hline Petritch & 15 & 9 & 1 & 5 \\
\hline Nevrokop & 36 & 25 & - & 11 \\
\hline Razlog & 9 & 4 & - & 5 \\
\hline Gorna Djoumaya & 14 & 5 & 1 & 8 \\
\hline TOTAL & 176 & 92 & 34 & 50 \\
\hline
\end{tabular}

Sources : ЦДА, Ф.176К, оП.2, А.Е.144, Л.153, « Tableau 2 », ci-joint à N. Semenov au MAЕ S. Paprikov, Serrès 4.11.1908, nº 609 ; ГЕОРГИЕВ - ТРИФОНОВ 1995, p. 576. Le consul grec à Serrès et le journal athénien E $\mu \pi \rho o ́ \varsigma ~(22.10 .1908$, p. 3) donnent des résultats légèrement différents. D'après le premier, on a élu au total 179 électeurs : 87 « ottomans » (c.-à-d. musulmans), 50 « Bulgares », 41 « Grecs » et un israélite (IAYE 1908/101, A. Saktouris au MAE, Serrès 24.10.1908, $n^{\circ}$ 6521). Selon le second, le nombre total des électeurs était 182, parmi lesquels 86 « Turcs », 51 « Bulgares » et 45 « Grecs ».

\section{Sandjak de Drama}

\begin{tabular}{|l|l|l|l|}
\hline \multirow{2}{*}{ KAZA } & \multicolumn{3}{|l|}{ Électeurs de $2^{\mathrm{e}}$ degré } \\
\cline { 2 - 4 } & TOTAL & Musulmans & « Grecs » \\
\hline Drama & 26 & 19 & 5 \\
\hline Kavala & 14 & 9 & 5 \\
\hline Pravi & 11 & 5 & 6 \\
\hline Šari Saban & 7 & 6 & 1 \\
\hline
\end{tabular}




\begin{tabular}{|l|l|l|l|}
\hline Ruptchuk & 8 & 8 & - \\
\hline TOTAL & 66 & 49 & 1 \\
\hline
\end{tabular}

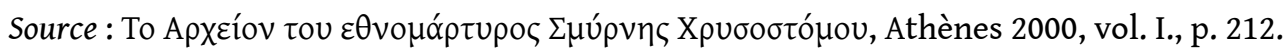

\section{Sandjak de Servia (Serfidje)}

\begin{tabular}{|l|l|l|l|l|}
\hline \multirow{2}{*}{ KAZA } & \multicolumn{4}{|l|}{ Électeurs de $2^{\text {e degré }}$} \\
\cline { 2 - 5 } & TOTAL & Musulmans & Grecs & Bulgares \\
\hline Servia & 11 & 2 & 9 & - \\
\hline Elassona & 13 & - & 13 & - \\
\hline Kozani & 18 & 10 & 8 & - \\
\hline Grevena & 24 & 3 & 21 & - \\
\hline Kaïlar & 18 & 16 & 1 & 1 \\
\hline Anaselitsa & 17 & 6 & 11 & - \\
\hline TOTAL & 102 & 38 & 63 & 1 \\
\hline
\end{tabular}

Source : IAYE 1908/101, L. Enyalis au MAE, Elassona 14/27.10.1908, No. 6210, 16/29.10.1908, $n^{\circ} 6270$ et 3.11.1908, n 598. Les mêmes numéros sont publiés dans la presse athénienne de l'époque (E $\mu \pi \rho o ́ \varsigma ~ 16.10 .1908)$. Le point d'interrogation dans le kaza de Kailar se rapporte à un électeur de $2^{\mathrm{e}}$ degré, "chrétien de Palaiohori » (village slavophone, aujourd'hui appelé Foufas), qui ne faisait pas partie des « nôtres », mais qui pendant le $2^{\mathrm{e}}$ tour a voté pour le bulletin jeune-turc.

\section{Sandjak de Monastir}

\section{Version grecque :}

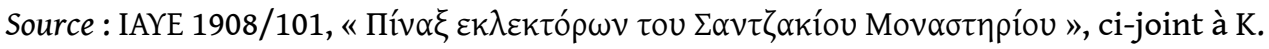
Dimaras au MAE, Monastir 30.10.1908, $\mathrm{n}^{\circ} 1207$.

\begin{tabular}{|l|l|l|l|l|l|}
\hline \multirow{2}{*}{ KAZA } & \multicolumn{4}{|l|}{ Électeurs de $2^{\text {e }}$ degré } \\
\cline { 2 - 6 } & TOTAL & Musulmans & Grecs & Bulgares & Serbes \\
\hline Monastir & 52 & 23 & 11 & 18 & - \\
\hline Ohrid & 25 & 9 & 1 & 15 & - \\
\hline Prilep & 31 & 12 & 3 & 13 & 3 \\
\hline Kičevo & 17 & 10 & - & 3 & 4 \\
\hline
\end{tabular}




\begin{tabular}{|l|l|l|l|l|l|}
\hline Florina & 16 & 8 & 5 & 3 & - \\
\hline TOTAL & 141 & 62 & 20 & 52 & 7 \\
\hline
\end{tabular}

\section{Version bulgare :}

Source : ЦДА, Ф.176К, оП.2, А.Е.144, Л.139-140, Т. Nedkov au MAЕ S. Paprikov, Monastir $31.10 .1908, \mathrm{n}^{\circ} 806$.

\begin{tabular}{|l|l|l|l|l|l|l|}
\hline \multirow{2}{*}{ KAZA } & \multicolumn{5}{l|}{ Électeurs de $2^{\text {e }}$ degré } \\
\cline { 2 - 7 } & TOTAL & Musulmans & Grecs & Bulgares & Serbes & Israélites \\
\hline Monastir & 57 & 23 & 11 & 21 & - & 2 \\
\hline Ohrid & 25 & 10 & - & 15 & - & - \\
\hline Prilep & 28 & 11 & - & 13 & 4 & - \\
\hline Kičevo & 17 & 11 & - & 3 & 3 & - \\
\hline Florina & 16 & 9 & 4 & 3 & - & - \\
\hline TOTAL & 143 & 64 & 15 & 55 & 7 & 2 \\
\hline
\end{tabular}

\section{Sandjak de Korçe}

\begin{tabular}{|l|l|l|l|l|l|}
\hline KAZA & TOTAL & Musulmans & Grecs & Bulgares & Roumains \\
\hline Korçë & 35 & 22 & 12 & - & 1 \\
\hline Kastoria & 21 & 9 & 6 & 6 & - \\
\hline Kolonia & 8 & 7 & 1 & - & - \\
\hline Starovo & 7 & 7 & - & - & - \\
\hline TOTAL & 71 & 45 & 19 & 6 & 1 \\
\hline
\end{tabular}

\section{Sandjak de Skopje}

\begin{tabular}{|l|l|l|l|l|}
\hline KAZA & TOTAL & Musulmans & « Bulgares » & « Serbes » \\
\hline Skopje & 45 & 34 & 11 & - \\
\hline Kumanovo & 23 & 8 & 10 & 5 \\
\hline Kratovo & 10 & 2 & 8 & - \\
\hline
\end{tabular}




\begin{tabular}{|l|l|l|l|l|}
\hline Radovič & 12 & 5 & 7 & - \\
\hline Stip & 17 & 8 & 9 & - \\
\hline Kočana & 16 & 8 & 8 & - \\
\hline Palanka & 13 & 2 & 6 & 5 \\
\hline Veles & 22 & 10 & 11 & 1 \\
\hline Pehčevo & 8 & 4 & 4 & - \\
\hline TOTAL & 174 & 85 & 78 & 11 \\
\hline
\end{tabular}

Source : ЦДА, Ф.176К, оП.2, А.Е.144, л.114 \& 122, I. Ikonomov au MAЕ S. Paprikov, Skopje 21.10.1908, no 694 , et 25.10.1908, no 707 .

\section{NOTES}

1. IAYE 1908/122, E. Kanellopoulos au MAE, Salonique 22.7.1908, no. 689; Robert Devereux, The first Ottoman Constitutional period, Baltimore, 1963; Hasan Kayali, "Elections and the Electoral Process in the Ottoman Empire, 1876-1919”, International Journal of Middle East Studies, 27 (1995), pp. 266-7.

2. Très emblématique est par exemple son traitement par le professeur grec Basil Gounaris : dans un chapitre de 21 pages dédié aux événements de la décennie 1903-1912, il n’y a aucune référence au déroulement ou aux résultats des élections; il mentionne seulement « la désillusion " produite

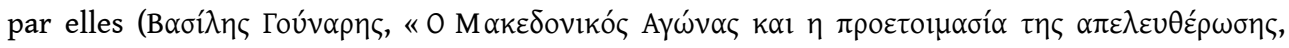

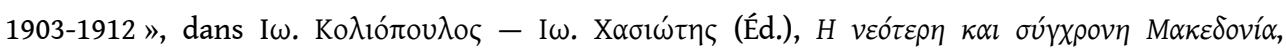
Athènes-Salonique, 1992, vol. I, p. 525). Evangelos Kofos, le doyen de l'historiographie grecque officielle sur la question macédonienne, évite de son côté toute mention à l'institution

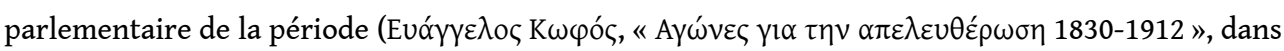

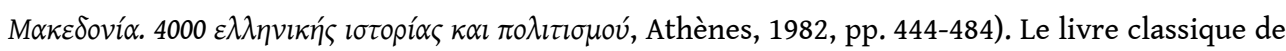
Douglas Dakin, publié à Salonique par l'Institut semi-officiel IMXA et considéré comme faisant partie de l'historiographie grecque, ne consacre aux élections de 1908 qu'une page et demie sur 538, sans la moindre référence bibliographique (Douglas Dakin, The Greek Struggle in Macedonia 1897-1913, Salonique, 1966, pp.391-392). Même l'ouvrage de Konstantinos Vakalopoulos, dédié exclusivement à la période Jeune-turque, écarte en substance le sujet : on n'y trouve que moins de trois pages (sur 452), qui reproduisent essentiellement le contenu du livre de Nikolaos

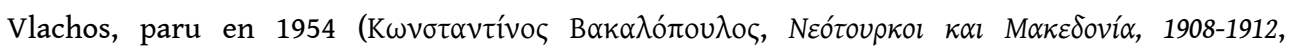
Salonique, 1988, pp. 241-244). Ce dernier est le seul à avoir accordé aux élections de 1908 une

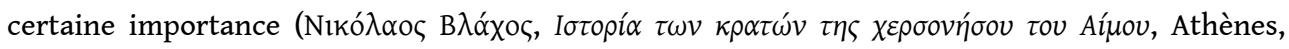
1954, pp. 137-144). En ce qui concerne l'historiographie de la République de la Macédoine exyougoslave, on note surtout les références trop laconiques -- ou même inexistantes -- aux élections de 1908 dans les récits officiels publiés par l'Institut d'Histoire Nationale : Институт ЗА НАЦИОНАЛНА ИСТОРИјА, ИСТОРИјА НА МАКЕДОНСКИОТ НАРОД, Skopje, 1969, vol. II, p. 302 ; Institut of National History, A history of the Macedonian people, Skopje, 1979; Institut of National History, History of the Macedonian people, Skopje, 2008, p. 200. 
3. Stojan Makedonski, « La révolution Jeune-Turque et les premières élections parlementaires de 1908 en Macédoine et en Thrace Orientale», Études Balkaniques, 4 (1974), pp. 133-146. Au contraire, l'ouvrage collectif plus récent de l'Institut Scientifique Macédonien omet complètement la question (МАКЕДОНСКИ НАУЧЕН ИНСТИТУТ, НАЦИОНАЛНО-ОСВОБОДИТЕЛНОТО ДВИЖЕНИЕ НА МАКЕДОНСКИТЕ И ТРАКИЙСКИТЕ БЪЛГАРИ, vol. III, Sofia, 1997.

4. Cf. la considération d'un ancien diplomate britannique, bon connaisseur du terrain : "In one sense, a race in Macedonia is merely a political party" (Charles Eliot, Turkey in Europe, $2^{\mathrm{e}}$ éd., Londres, 1908, p. 271). La même terminologie est très couramment utilisée par tous les acteurs de

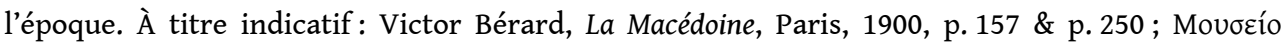

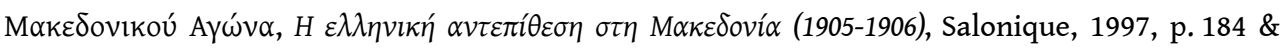

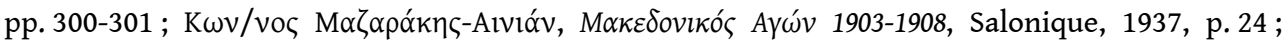

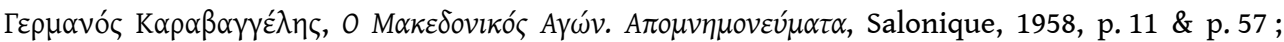
АТАНАС ШОПОВ, ИЗ ЖИВОТА И ПОЛОЖЕНИЕТО НА БЪЛГАРИТЕ В ВИЛАЕТИТЕ, Plovdiv, 1893, pp. 68, 70, 85 \& 232 ; ГЕОРГИ БАЖДАРОВ, ГОРНО-БРОДИ, Sofia, 1929, pp. 41, 44, 59-60 \& 62 ; ВАСИЛ КЪНЧОВ, МАКЕДОНИЯ. ПЪТЕПИСИ, Sofia, 2000, pp. 187 \& 451 ; Jovan Cvijić, Questions Balkaniques, Paris-Neuchatel, 1917, pp. 74 \& 78-79.

5. IAYE 1904/74, L. Koromilas au MAE, Salonique, 17.12.1904, No. 46 Special ; Albert Sonnichsen, Confessions of a Macedonian Bandit, N. York, 1909; ВЕЛИЧКО ГЕОРГИЕВ - СТАЙКО ТРИФОНОВ, ГРЪЦКАТА И СРЪБСКАТА ПРОПАГАНДИ В МАКЕДОНИЯ, Sofia, 1995, p. 111; The University 'Cyril and Methodius', Documents on the Struggle of the Macedonian People for Independence and a Nation-State, Skoje, 1985, vol. I, pp. 529-535 ; Tchavdar Marinov, «L'impasse du Passé. La Construction de l'identité nationale macédonienne et le conflit politico-historiographique entre la Bulgarie et la Macédoine ", Thèse doctorale à l'EHESS, Paris, 2006, pp. 89-143. Pour le terme "parti macédonien »: Rudolf Reiss, Rapport sur la situation des bulgarophones et des musulmans dans les nouvelles provinces grecques, s.l. 1915, p. 105.

6. Pour le cas albanais : Nathalie Clayer, Aux origines du nationalisme albanais, Paris, 2008.

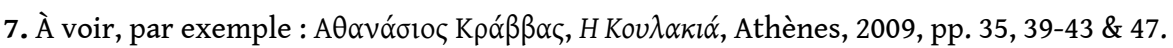

8. Fikret Adanır, Die Makedonische Frage, Wiessbaden, 1979 ; ХРИСто СИлЯнов, освоБОДИТЕЛниТЕ

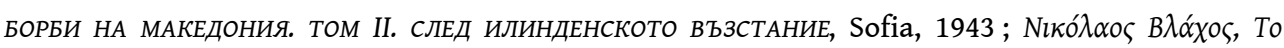

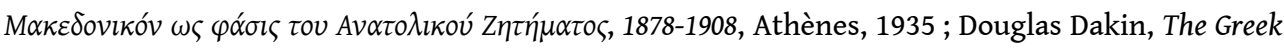
struggle in Macedonia, 1897-1913, Salonique, 1966.

9. IAYE 1907/14, Е. Zalokostas au MAE, Sofia, 22.6.1907, no.613; АЛЕКСО СТЕФАНОВ, РЕВОЛЮЦИОННАТА ДЕЙНОСТ В ДЕМИРХИСАР (БИТОЛСКО), Sofia, 1931, pp.14-15; Вабі́

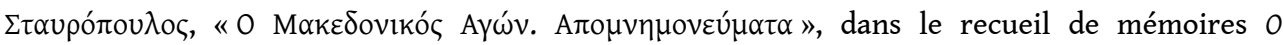

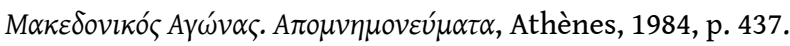

10. Clayer, 2008, pp. 561-572.

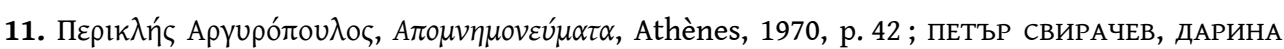
ИЛИЕВА, « ПОВЕРИТЕЛЕН ДОКЛАД ОТ АНДРЕЙ ТОШЕВ ПО МАКЕДОНСКИЯ ВЪПРОС ОТ 1907 Г. », ИЗВЕСТИЯ НА ДЪРЖАВНИТЕ АРХИВИ, 64 (1992), pp. 159-161.

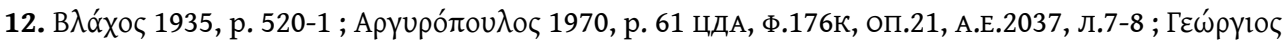

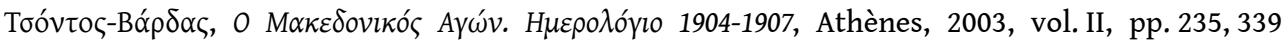

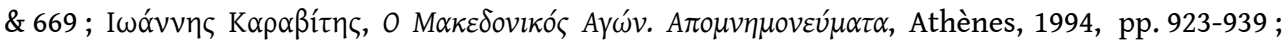
КИРИЛ ПЪРЛИЧЕВ, КЮСТЕНДИЛСКИЯТ КОНГРЕС НА ВМРО, 1908 Г., Sofi,a 2001, pp. 27, 66 \& 73; ВЛАДИМИР КАРАМФИЛОВ, СПОМЕНИ ЗА ПРОСВЕТНОТО ДЕЛО И РЕВОЛЮЦИОННИТЕ БОРБИ В ГР. ПРИЛЕП, Sofia, 2005, p. 72. Pour le terme « xénélasie » : ГАК/АТВ/13, rapport manuscrit de G. Vardas, écrit

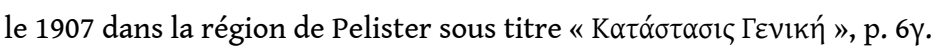

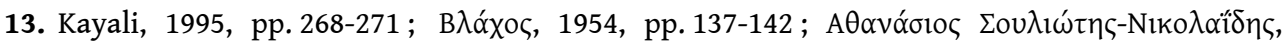

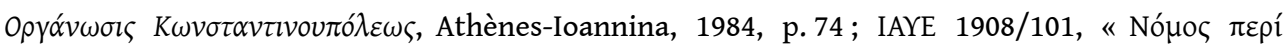

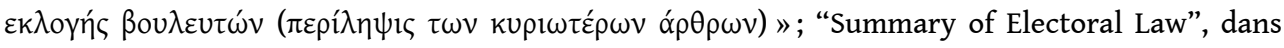


Turkey, $\mathrm{n}^{\circ} 1$ (1909). Correspondence Respecting the Constitutional Movement in Turkey, 1908, Londres, 3.1909, Cd. 4529 [Blue Book 1 (1909)], p. 102; цДА, Ф.176К, оП.2, А.Е.144, Л.120-1; цДА, Ф.176К, оП. 2, А.Е.144, л.82, Ikonomov au MAE S. Paprikov, Skopje, 4.9.1908, No. 595.

14. IAYE 1908/101, E. Kanellopoulos au MAE G. Baltatzis, Salonique, 30.7.1908, no. 719; IAYE 1908/59, A. Chalkiopoulos au MAE G. Baltatzis, Monastir, 1.9.1908, no. 980; IAYE 1908/101, Giannopoulos au MAE, Serrès 18.8.1908, no. 416; IAYE 1908/92, I. Argyropoulos au MAE G. Baltatzis, Belgrade, 2.8.1908, n ${ }^{\circ}$ 105; ВЕЛИЧКО ГЕОРГИЕВ - СТАЙКО ТРИФОНОВ (eds), МАКЕДОНИЯ И ТРАКИЯ В БОРБА ЗА СВОБОДА, Sofia, 1995, pp. 553-554 \& 557; цДА, Ф.176К, ОП.2, А.Е.144, Л.83-84 \& 95, T. Nedkov au MAE S. Paprikov, Monastir, 10.9.1908, no. 679 et 25.9.1908, no. 723; цДА, Ф. 176К, оП.2, А.Е.144, л.53-54 \& 74-77, Ikonomov au MAE S. Paprikov, Skopje, 6.8.1908, no. 553 et 27.8.1908, no. 466 .

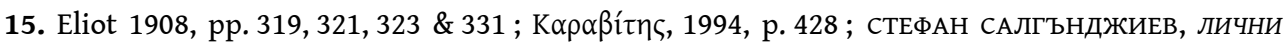
ДЕЛА И СПОМЕНИ ПО ВЪЗРАЖДАНЕТО НА СОЛУНСКИТЕ И СЕРСКИ БЪЛГАРИ, Plovdiv, 1906, pp. 32-33 \& 65 ; ГЕОРГИ ТРАЙЧЕВ, СПОМЕНИ ОТ МОЕТО 40 ГОДИШНО УЧИТЕЛСТВО, Sofia, 1930, p. 23 ; ИВАН СНЕГАРОВ, СОЛУН В БЪЛГАРСКАТА ДУХОВНА КУЛТУРА, Sofia, 1937, p. 116.

16. IAYE 1908/92, I. Argyropoulos au MAE G. Baltatzis, Belgrade, 30.7.1908, no. 101; IAYE 1908/101, Giannopoulos au MAE, Serrès 18.8.1908, no. 416.

17. Cela, malgré le fait que ськк a officiellement condamné l'indépendance bulgare et participé aux manifestations organisées par les Jeunes-Turcs pour la dénoncer : IAYE 1908/59, Gogos au MAE, Monastir 26.9.1908, No. 5549 et 27.9.1908, №. 5555 ; IAYE 1908/59, K. Dimaras au MAE, Monastir, 3.10.1908, no. 1093 ; СБКК, ДНЕВНИЦИ НА УЧРЕДИТЕЛНИЯ И ВТОРИЯ КОНГРЕСИ, Salonique, 1910, pp. 27-28.

18. IAYE 1908/99, K. Dimaras au MAE, Monastir, 14/27.9.1908, no. 5032, 2/15.10.1908, no. 5825 et 4.10.1908, no. 1084. Gogos était élu Gogos comme candidat le 13 Septembre, par une réunion de la communauté "grecque» (patriarchiste) de la ville élargie avec la participation de 29 autres citadins ; il avait obtenu 14 voix contre 8 pour l'avocat Tsigaras, représentant de la communauté à la Commission Mixte.

19. IAYE 1908/122, A. Papadiamantopoulos au MAE, Salonique, 12.9.1908, no. 894 ; IAYE 1908/99, le même au MAE G. Baltatzis, Salonique, 14.9.1908, no. 900, 18.9.1908, no. 915 et 20.9.1908, no. 928. Honaios avait formellement démissionné de son service au consulat, sans être privé de son salaire (ÉAYE 1908/59, Papadiamantopoulos à G. Baltatzis, Salonique, 11.11.1908, no. 1091).

20. СБКК 1910, pp. 29-32 ; цДА, Ф.176К, ОП.2, А.Е.144, Л.101-102, I. Ikonomov au MAE S. Paprikov, Skopje, 3.10.1908, no. 658.

21. IAYE 1908/101, L. Enyalis au MAE, Elassona, 26.9.1908, no. 520.

22. IAYE 1908/101, Kavallieratos au MAE, Kavalla, 6.10.1908, no. 709 .

23. B $\lambda$ óxOৎ 1954, p. 143; Sir G. Lowther, “Annual Report on Turkey for thw Year 1908”, dans G.P. Gooch - H. Temperley, British Documents on the Origins of the War, 1898-1914, vol. V., The Near East, Londre,s 1928, р. 279; цДА, Ф.176К, оП.2, А.Е.144, л.23-31, Bureau Central des Clubs Constitutionnels Bulgares, «Les élections et les Bulgares Ottomans »; ЦДА, Ф.176К, ОП.2, А.Е.144, Л.114, I. Ikonomov au MAE S. Paprikov, Skopje, 21.10.1908, no. 694.

24. IAYE 1908/101, Kavallieratos au MAE, Kavalla, 5.8.1908, no. 536, et 8/21.9.1908, no. 4947 ; Lamb à Lowther, Salonique, 22.9.1908, dans Blue Book 1 (1909), p. 94.

25. IAYE 1908/101, A. Papadiamantopoulos au MAE G. Baltatzis, Salonique, 27.10.1908, no. 1052 ; ДАНЧО ЗОГРАФСКИ, АВСТРИСКИ ДОКУМЕНТИ ЗА ИСТОРИјАТА НА МАКЕДОНСКИОТ НАРОД. ТОМ II (1907-1908), Skopje, 1981, p. 241.

26. IAYE 1908/101, A. Papadiamantopulos au MAE G. Baltatzis, Salonique, 22.9.1908, no. 931 ; Lamb a Lowther, Salonique, 22.9.1908, dans Blue Book 1 (1909), p. 95.

27. IAYE 1908/101, А. Papadiamantopoulos au MAE G. Baltatzis, no. 994 ; цДА, Ф.176К, ОП.2, А.Е.

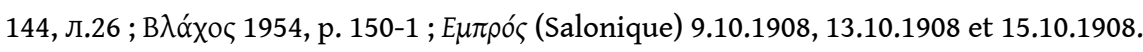


28. IAYE 1908/101, A. Papadiamantopoulos au MAE G. Baltatzis, no. 994.

29. IAYE 1908/101, A. Papadiamantopoulos au MAE G. Baltatzis, Salonique, 27.10.1908, no. 1051 et 1052 ; СБКК 1910, p. 28 ; ГЕОРГИЕВ - ТРИФОНОВ 1995, p. 566.

30. ДИМИТАР ВЛАХОВ, МЕМОАРИ, Skopje1970, p. 87-8.

31. IAYE 1908/101, A. Papadiamantopoulos au MAE G. Baltatzis, Salonique 5.9.1908, no. 870. Cf. ЙОРДАН ПОП ГЕОРГИЕВ, СТ.ШИШКОВ, БЪЛГАРИТЕ В СЕРСКОТО ПОЛЕ, Plovdiv, 1918, pp. 31-2.

32. ЦДА, Ф.176К, ОП.2, А.Е.144, Л.85-86, N. Semenov au MAЕ S. Paprikov, Serrès 15.9.1908, no. 539. Pour la réaction du consul grec de la ville : ÉAYE 1908/99, Saktouris au MAE., Serrès 10/23.9.1908, no. 4964.

33. IAYE 1908/71, А. Saktouris au MAE., Serrès 27.7.1908, nо. 3782 ; цДА, Ф.176К, оП.2, А.Е.144, л. 148-9, N. Semenov au MAE S. Paprikov, Serrès 4.11.1908, no. 609.

34. IAYE 1908/99, А. Saktouris au MAE., Serrès 28.9.1908, no. 5549; ГЕОРГИЕВ ТРИФОНОВ 1995, p. 577.

35. IAYE 1908/99, А. Saktouris au MAE., Serrès 28.9.1908, no. 5549 ; ГЕОРГИЕВ ТРИФОНОВ 1995, p. 577.

36. ЦДА, Ф.176К, ОП.2, А.Е.144, Л.151; ГЕОРГИЕВ - ТРИФОНОВ 1995, pp. 574-575.

37. ЦДА, Ф.176К, оП.2, А.Е.144, л.153, « Tableau 2 », ci-joint à N. Semenov au MAE S. Paprikov, Serrès 4.11.1908, no. 609 ; ГЕОРГИЕВ ТРИФОНОВ 1995, б.576. Le consul grec à Serrès a rapporté des résultats légèrement différents : 86 "Turcs », 51 "Bulgares », 41 "Grecs » et 1 Israélite (IAYE 1908/101, Saktouris au MAE, Serrès 24.10.1908, no. 6521).

38. ЦДА, Ф.176К, ОП.2, А.Е.144, Л.154; ГЕОРГИЕВ ТРИФОНОВ 1995, б.577.

39. IAYE 1908/101, A. Saktouris au MAE, Serrès 24.10.1908, $n^{\circ} 6521$. Il est probable que le consul confond les deux tours, étant donné qu'au kaza de Melnik il n'y avait pas des électeurs de deuxième degré Grecs.

40. цДА, Ф.176К, оп.2, А.Е.144, л.158-161, N. Semenov au MAЕ S. Paprikov, Serrès, 7.11.1908, no. 615.

41. ПАВЕЛ ДЕЛИРАДЕВ, ЯНЕ САНДАНСКИ, Sofia 1946 ; АНГЕЛ ДИНЕВ, ИЛИНДЕНСКАТА ЕПОПЕјА, Skopje 1987 ; ИВА БУРИЛКОВА - ЦОЧО БИЛЯРСКИ, «ПРЕДГОВОР ", dans le recueil ЯНЕ САНДАНСКИ. СПОМЕНИ, Sofia, 2007, p. 5-14.

42. Mercia MacDermott, For freedom and perfection: the life of Yane Sandansky, Londres 1988, pp. 371-372 \& 381.

43. MacDermott 1988, pp. 371-372 \& 380; ГЕОРГИЕВ ТРИФОНОВ 1995, pp. 505-506, 553-554 \& 565.

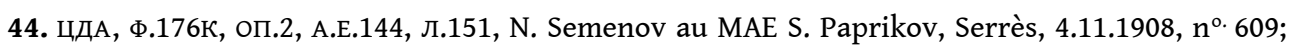
цДА, Ф.176К, оП.2, А.Е.144, л.155-156,. А. Šopov au MAЕ S. Paprikov, Salonique, 6.11.1908, $\mathrm{n}^{\text {o. }} 1134$. Pour les ordres du Ministère: ГЕОРГИЕВ ТРИФОНОВ 1995, pp. 506-507; цДА, Ф.176К, ОП.2, А.Е.144, Л.118-119, S. Paprikov à A. Šopov, Sofia, 4.10.1908, no. 1261.

45. Lamb à Lowther, Salonique, 9.11.1908, dans Blue Book 1 (1909), p. 101 ; IAYE 1908/101, A. Saktouris au MAE, Serrès, 15.10.1908, no. 6242.

46. ЦДА, Ф.ЗК, ОП.8, А.Е.1213, Л.85Б, S. Radev à N. Genadiev, Salonique, s.d. [8.1908], sur l'activité du consul français à Salonique, baron de Steeg. Cf aussi les rapports du consul de la Russie dans la même ville, К.А. Kochmanski : СИМОН ДРАКУЛ (ed.), МАКЕДОНИјА МЕЃУ АВТОНОМИјАТА И ДЕЛЕЖОТ. ЗБОРНИК РУСКА ДИПЛОМАТСКА ДОКУМЕНТАЦИјА -1894-1913, vol. VI, Skopje, 2006, pp. 431-438.

47. IAYE 1908/100, K. Dimaras au MAE, Monastir, 17.9.1908, no. 5149.

48. IAYE 1908/99, K. Dimaras au MAE, Monastir 11.9.1908, no. 1022; IAYE 1908/59, A. Chalkiopoulos au MAE G. Baltatzis, Monasti,r 1.9.1908, no. 980.

49. IAYE 1908/99, A. Chalkiopoulos au MAE G. Baltatzis, Monastir 1.9.1908, no. 976 ; цДА, Ф.176К, оп.2, А.Е.144, л.83-84 \& 95, Т. Nedkov au MAE S. Paprikov, Monastir 10.9.1908, no. 679, et 25.9.1908, no. 723 . 
50. IAYE 1908/99, К. Dimaras au MAE, Monastir 11.9.1908, no. 1022, et 20.9.1908, no. 1039 ; цДА, Ф. 176К, оП.2, А.Е.144, л.112-113 \& 142, Т. Nedkov au MAE S. Paprikov, Monastir 20.10.1908, no. 777, et 2.11 .1908$, no. 811 .

51. IAYE 1908/99, K. Dimaras au MAE, Monastir 14/27.10.1908, no. 6241.

52. СБКК 1910, б.29; цДА, Ф.176К, ОП.2, А.Е.144, Л.142-143, Т. Nedkov au MAE S. Paprikov, Monastir 2.11.1908, no. 811.

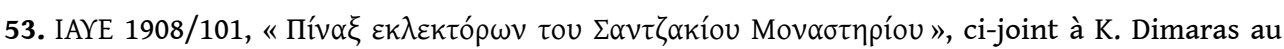
MAE, Monastir 30.10.1908, nо. 1207 ; ЦДА, Ф.176К, оП.2, А.Е.144, Л.139-140, Т. Nedkov au MAE S. Paprikov, Monastir 31.10.1908, no. 806. Sur les trucs electoraux elaborés contre le parti bulgare, la сБкК a composé un dossier très détaillé : цДА, Ф.176К, оп.2, А.Е.144, л.23-31, Bureau Central des Clubs Constitutionnels Bulgares, «Les élections et les Bulgares Ottomans ».

54. ЦДА, Ф.176К, ОП.2, А.Е.144, л.30-31.

55. IAYE 1908/99, K. Dimaras au MAE, Monastir 20.9.1908, no. 1039, 4.10.1908, no. 1084, et 14.10.1908, no. 1141 .

56. ЦДА, Ф.176К, ОП.2, А.Е.144, Л.103, Т. Nedkov au MAЕ S. Paprikov, Monastir 8.10.1908, no. 717.

57. ЦДА, Ф.176К, оП.2, А.Е.144, Л.112, Т. Nedkov au MAE S. Paprikov, Monastir 20.10.1908, no. 777.

58. ЦДА, Ф.176К, ОП.2, А.Е.144, Л.162, Т. Nedkov au MAE, Monastir 7.11.1908, no. 820.

59. IAYЕ 1908/101, К. Dimaras au MAE, Monastir 30.10.1908, nº 1207, et 8.11.1908, no. 1222 ; ЦДА, Ф.176К, ОП.2, А.Е.144, л.142.

60. IAYE 1908/101, A. Papadiamantopoulos au MAE G. Baltatzis, Salonique 5.9.1908, no. 870. Cf. ЙОРДАН ПОП ГЕОРГИЕВ, СТ.ШИШКОВ, БЪЛГАРИТЕ В СЕРСКОТО ПОЛЕ, Plovdiv, 1918, pp. 31-32.

61. IAYE 1908/99, K. Dimaras au MAE, Monastir 11.9.1908, no. 1022; IAYE 1908/100, A. Papadiamantopoulos au MAE, Salonique 11/24.11.1908, no. 7006. Pour les propositions pareilles antérieures concernant les sandjaks de Serrès et de Monastir : IAYE 1908/99, Saktouris au MAE, Serrès 28.9.1908, no. 5549 ; IAYE 1908/100, K. Dimaras au MAE, Monastir 16/29.9.1908, no. 5131, et 17/30.9.1908, no. 5149 ; IAYE 1908/99, K. Dimaras au MAE, Monastir 20.9.1908, no. 1039.

62. IAYE 1908/99, K. Dimaras au MAE, Monastir 11.9.1908, no. 1022,. 4.10.1908, no. 1084, et 22.10.1908, no. 1159 .

63. IAYE 1908/99, K. Dimaras au MAE, Monastir 3/16.11.1908, no. 6803, et 8.11.1908, no. 1222.

64. IAYE 1908/101, A. Papadiamantopoulos au MAE G. Baltatzis, Salonique 5.9.1908, no. 870 ; ЦДА, Ф.176К, оП.2, А.Е.144, л.53-54, І. Ikonomov au MAE S. Paprikov, Skopje 6.8.1908, no. 553.

65. ЦДА, Ф.176К, ОП.2, А.Е.144, л.74-77, I. Ikonomov au MAЕ S. Paprikov, Skopje 27.8.1908, no. 466. 66. ЦДА, Ф.176К, оП.2, А.Е.144, л.114 \& 122, I. Ikonomov au MAЕ S. Paprikov, Skopje 21.10.1908, no. 694, et 25.10.1908, no. 707 ; IAYE 1908/101, N. Baraklis au MAE, Skopje 18/31.10.1908, no. 6359. 67. СБКК 1910, р. 29 ; цДА, Ф.176К, ОП.2, А.Е.144, Л.124-127 \& 141, I. Ikonomov au MAE S. Paprikov, Skopje 27.10.1908, $\mathrm{n}^{\circ} 709$, et 1.11.1908, $\mathrm{n}^{\circ} 717$; IAYE 1908/101, N. Baraklis au MAE, Skopje 2/21.11.1908, no. 6975 .

68. ЦДА, Ф.176К, ОП.2, А.Е.144, Л.100-101, Ikonomov au MAЕ S. Paprikov, Skopje 3.10.1908, no. 658.

69. ЦДА, Ф.176К, ОП.2, А.Е.144, Л.100, Ikonomov au MAE S. Paprikov, Skopje 3.10.1908, no. 658.

70. IAYE 1908/99, N. Baraklis au MAE, Skopje 29.10.1908, no. 595.

71. Sükrü Hanioglu, “The Second Constitutional Period, 1908-1918”, dans Reşat Kasaba (ed.), The Cambridge History of Turkey, vol. 4, Cambridge, 2008, p.67; Kayali 1995, p. 271. voir aussi: Feroz Ahmad, The Young Turks, Oxford, 1969, p. 27-8.

72. Lamb à Lowther, Salonique 9.11.1908, et Lowther à Sir Edward Grey, Therapia 9.10.1908, dans Blue Book 1 (1909), pp. 101 \& 93; “Annual Report for Turkey for 1908”, op.cit. p. 279; A. Rapport à Aehrenthal, Salonique 6.11.1908, no. 114, dans ЗОГРАФски 1981, p. 239.

73. Cf. par exemple la lettre personnelle (31.7.08) du ministre anglais des affaires extérieures, Sir Edward Grey, à son ambassadeur à Constantinople (Gooch - Temperley 1928, p. 263). 


\section{RÉSUMÉS}

Les élections parlementaires de 1908 ont été la première expérience de cette sorte pour la population ottomane, la première épreuve de la crédibilité des promesses faites par les JeunesTurcs, un premier test pour les partis «nationaux ». Les résultats de ces élections à deux tours sont loin de refléter l'opinion publique : négociations entre les partis nationaux et les JeunesTurcs pour obtenir leur soutien contre un autre parti national, jeu des Jeunes-Turcs qui soutiennent en général le plus faible localement contre ses rivaux, trucages électoraux variés. Chacun des partis néanmoins dans son historiographie nationale se pose en victime en oubliant ses propres manœuvres. Le recours aux Archives permet d'en appréhender l'étendue.

The 1908 general election was the first experience of this kind for the ottoman population, the first test for the credibility of fine promises made by the Young Turks and the first test for the "national" party. The results of these two rounds election are very far from reflecting the public opinion: bargain between the national parties and the Young Turks to gain their support against another national party, game of the Young Turks who, as a rule, support the locally weakest against his contenders, various election fixing. Each party nevertheless in his national historiography, presents itself as a victim while forgetting its own fixing. Using the public records and government archives makes it possible to go deeper in the subject.

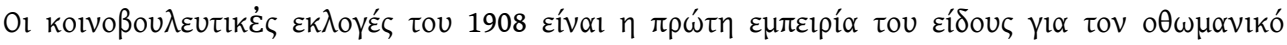

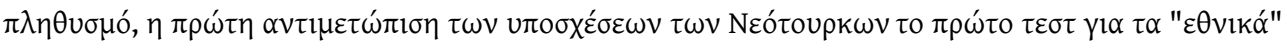

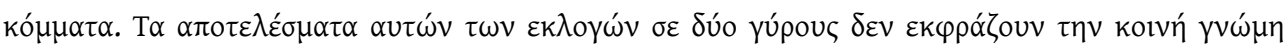

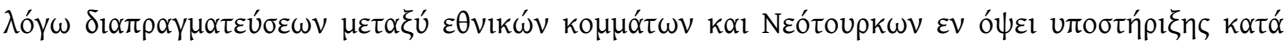

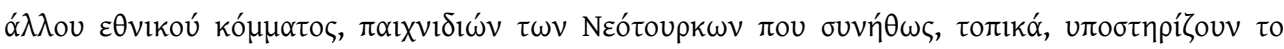

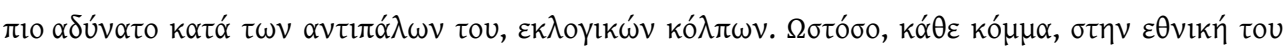

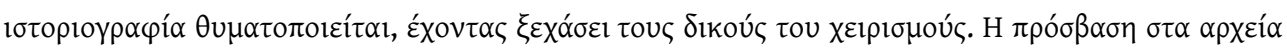

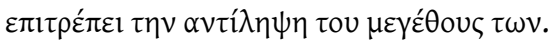




\section{INDEX}

Index géographique : Macédoine, Salonique, Servia, Skopje, Zihna, Debar, Demir Hissar, Drama, Ohrid, Edessa, Gorna Djoumaïa, Istanbul, Korçe, Nevrokop, Serrès

Index chronologique : révolution jeune-turque (1908-1914)

Thèmes : Histoire, Sciences politiques

Mots-clés : CUP, Épitrope, élections, Esnaf, Jeunes-Turcs, Exarchiste/Patriarchiste, nation, Hilmi Pacha (1855-1922), nationalisme, Jeunes-Turcs, ottomanisme, Caza/Kaza, Mahalle, Millet, Muchtar, ORIM/VRMO, Pomak, Sandanski Yané (1872-1915), Sandjak, Tanzimat, Tchorbadji, Torbesh, Valaades, Valaque, Vilayet, Xénélasie

motsclesmk ИСТАНБУЛ, КОРЧА, МАКЕДОНИЈА, МЛАДОТУРСКАТА, НЕВРОКОП, ОХРИД, СКОПЈЕ, СОЛУН, СЕРВИА

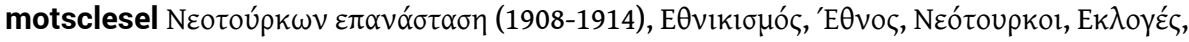

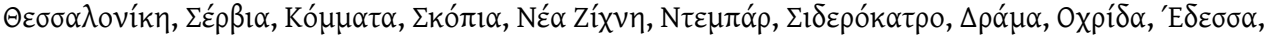

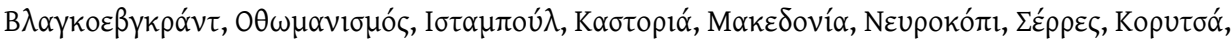
$\Sigma \alpha \lambda$ ovíkn

motsclestr Selanik, Servia, Üsküb, Nea Zichni, Debar, Demir Hissar, Drama, Ohrid, Edhessa, Blagoevgrad, İstanbul, Korça, Makedonya, Nevrokop, Serres, Genç Türk İhtilâli (1908-1914), Genç Türkler

Keywords : elections, nationalism, ottomanism, Salonika, Serres, Young Turks revolution (1908-1914), Skopje, Zihna, Debar, Demir Hissar, Drama, Ohrid, Edessa, Gorna Djumaïa, Politics, History, Nevrokop 